IJMMS 31:12 (2002) 725-729

PII. S0161171202108088

http://ijmms.hindawi.com

(c) Hindawi Publishing Corp.

\title{
A NOTE ON OPERATORS OF DELETION AND CONTRACTION FOR ANTICHAINS
}

\author{
ANDREY O. MATVEEV \\ Received 16 August 2001 and in revised form 28 March 2002
}

\begin{abstract}
The operators of deletion and contraction for clutters are generalized to those for antichains of finite bounded posets. A generalization of the result by Seymour (1976), describing the relationship between the operators of deletion, contraction, and the blocker map, is considered as a comparison in the lattice of antichains of a poset.
\end{abstract}

2000 Mathematics Subject Classification: 06A06, 90C27.

1. Introduction. Deletion and contraction are basic operators on clutters. Recall that for a finite nonempty set $S$, a family of its subsets is called a clutter (or a Sperner family) if no set from that family contains another. Denote by 0 the empty subset of $S$. The clutter $\varnothing$ (containing no sets) and the clutter $\{\hat{0}\}$ are called trivial.

Consider a nontrivial clutter $\mathscr{G}$ on the ground set $S$. Let $x \in S$. Recall that the deletion $\varphi \backslash x$ and contraction $\varphi / x$ are the clutters, defined as follows:

$$
\begin{aligned}
& \mathscr{G} \backslash x=\{G \in \mathscr{G}: G \not \nexists x\}, \\
& \mathscr{G} / x=\{\text { inclusion-wise minimal sets of the family }\{G-\{x\}: G \in \mathscr{G}\},
\end{aligned}
$$

on the ground set $S-\{x\}$.

Deletion and contraction are also defined for the trivial clutters

$$
\varnothing \backslash x=\varnothing / x=\varnothing, \quad\{\hat{0}\} \backslash x=\{\hat{0}\} / x=\{\hat{0}\},
$$

on the ground set $S-\{x\}$.

If $X=\left\{x_{1}, \ldots, x_{n}\right\} \subseteq S, n \geq 1$, then the deletion $\mathscr{G} \backslash X$ and contraction $\mathscr{G} / X$ are defined in the following way: $\mathscr{G} \backslash X=\mathscr{G} \backslash x_{1} \backslash \cdots \backslash x_{n}$, and $\mathscr{G} / X=\mathscr{G} / x_{1} / \cdots / x_{n}$. Deletions and contractions, sequentially performed on a clutter, produce its minors.

The blocker of a nontrivial clutter $\mathscr{G}$ on the ground set $S$ is the clutter $\mathscr{B}(\mathscr{G})$ on $S$, consisting of all the inclusion-wise minimal subsets $H \subseteq S$ with the property, for each $G \in \varphi,|H \cap G| \geq 1$.

The blockers of the trivial clutters are defined as follows (see, e.g., [2]):

$$
\mathscr{B}(\{\hat{0}\})=\varnothing, \quad \mathscr{B}(\varnothing)=\{\hat{0}\} .
$$

Seymour described in [4] the relationship between a clutter $\mathscr{G}$ on the ground set $S$, deletions, contractions, and relevant blockers; if $X$ is a nonempty subset of $S$ then we have

$$
\mathscr{B}(\mathscr{G}) \backslash X=\mathscr{B}(\mathscr{G} / X), \quad \mathscr{B}(\mathscr{G}) / X=\mathscr{B}(\mathscr{G} \backslash X)
$$


Recall that the clutters on $S$ are in natural one-to-one correspondence with the antichains of the Boolean lattice of all subsets of $S$.

We present in this paper operators of deletion and contraction for antichains of a finite bounded poset. The main results of the paper are Theorems 2.5 and 2.6. Theorem 2.5 states that deletion and contraction for antichains are (co)closure operators on the lattice of antichains of the poset. Theorem 2.6 provides a generalization of result (1.4) to antichains of the poset.

It is a consequence of Theorem 2.6 that equalities (1.4) might be read as

$$
\mathscr{B}(\mathscr{G}) \backslash X=\mathscr{B}(\mathscr{G} / X) \preceq \mathscr{B}(\mathscr{G}) \preceq \mathscr{B}(\mathscr{G}) / X=\mathscr{B}(\mathscr{G} \backslash X),
$$

where $\preceq$ is a certain comparison that comes from the lattice of antichains of the Boolean lattice of all subsets of $S$.

2. Deletion and contraction. We refer the reader to [5, Chapter 3] for basic information and terminology in the theory of posets.

We use $\min Q$ to denote the set of all minimal elements of a poset $Q$. If $Q$ has a least element then it is denoted $\hat{0}_{Q}$; if $Q$ has a greatest element then it is denoted $\hat{1}_{Q}$.

Throughout this note, $P$ stands for a finite bounded poset with $|P|>1$; $P^{\text {a }}$ denotes its atom set, that is the set of all elements covering $\hat{0}_{P} \cdot \mathfrak{I}(A)$ and $\mathfrak{f}(A)$ denote the order ideal and filter of $P$ generated by an antichain $A$ of $P$, respectively.

We denote the distributive lattice of all antichains of $P$ by $\mathcal{H}(P)$. If $A_{1}, A_{2} \in \mathcal{H}(P)$ then we set

$$
A_{1} \leq A_{2} \quad \text { iff } \mathfrak{f}\left(A_{1}\right) \subseteq \mathfrak{f}\left(A_{2}\right) .
$$

The least and greatest elements $\hat{0}_{\mathcal{H}(P)}$ and $\hat{1}_{\mathcal{H}(P)}$ of $\mathcal{H}(P)$ are the trivial antichains $\varnothing \subset P$ and $\left\{\hat{0}_{P}\right\}$, respectively. For the rest of the paper, we denote by $\wedge$ and $\vee$ the operations of meet and join in the lattice $\mathcal{H}(P)$; if $A_{1}, A_{2} \in \mathcal{H}(P)$ then

$$
\begin{aligned}
& A_{1} \vee A_{2}=\min \left(A_{1} \cup A_{2}\right), \\
& A_{1} \wedge A_{2}=\min \left(\mathfrak{f}\left(A_{1}\right) \cap \mathfrak{f}\left(A_{2}\right)\right),
\end{aligned}
$$

respectively.

Let $A$ be a nontrivial antichain of $P$, that is $A \in \mathfrak{H}(P)-\left\{\hat{0}_{\mathfrak{H}(P)}, \hat{1}_{\mathcal{H}(P)}\right\}$. The blocker $\mathfrak{b}(A)$ of $A$, defined in [3], is the antichain

$$
\min \left\{b \in P:\left|\mathfrak{z}(b) \cap \mathfrak{I}(a) \cap P^{\mathrm{a}}\right| \geq 1 \forall a \in A\right\} .
$$

The blockers of the trivial antichains are defined as follows:

$$
\mathfrak{b}\left(\hat{0}_{\mathfrak{A}(P)}\right)=\hat{1}_{\mathfrak{A}(P)}, \quad \mathfrak{b}\left(\hat{1}_{\mathfrak{A}(P)}\right)=\hat{0}_{\mathfrak{A}(P)} .
$$

For a one-element antichain $\{a\}$ of $P$, we write $\mathfrak{b}(a)$ instead of $\mathfrak{b}(\{a\})$. If $a \neq \hat{0}_{P}$ then $\mathfrak{b}(a)=\mathfrak{I}(a) \cap P^{\mathrm{a}}$, and we have $\{a\} \leq \mathfrak{b}(\mathfrak{b}(a)) \leq \mathfrak{b}(a)$.

If $A$ is a nontrivial antichain of $P$ then its blocker $b(A)$ is determined, in particular, by the equality $\mathfrak{b}(A)=\bigwedge_{a \in A} \mathfrak{b}(a)$.

The map $\mathfrak{b}: \mathfrak{A}(P) \rightarrow \mathfrak{A}(P)$, reflecting an antichain to its blocker, is the blocker map on $\mathcal{A}(P)$; it is antitone. The composite map $\mathfrak{b} \circ \mathfrak{b}$ is a closure operator on $\mathcal{A}(P)$. The 
image $\mathfrak{b}(\mathcal{A}(P))$ is called in [3] the lattice of blockers in $P$ and it is denoted $\mathfrak{b}(P)$. The restriction $\left.\mathfrak{b}\right|_{\mathfrak{B}(P)}$ of the blocker map is an anti-automorphism of $\mathfrak{2}(P)$. The lattice $\mathfrak{B}(P)$ is a meet-subsemilattice of $\mathfrak{A}(P)$. For every blocker $B \in \mathfrak{Z}(P)$, its preimage $\mathfrak{b}^{-1}(B)$ is a convex join-subsemilattice of $\mathcal{A}(P)$; the greatest element of $\mathfrak{b}^{-1}(B)$ is $\mathfrak{b}(B)$.

We start with generalizing the notions of deletion and contraction.

DEFINITION 2.1. Let $X \subseteq P^{\mathrm{a}},|X| \geq 1$.

(i) If $\{a\}$ is a nontrivial one-element antichain of $P$ then the deletion $\{a\} \backslash X$ and contraction $\{a\} / X$ are the antichains

$$
\begin{aligned}
& \{a\} \backslash X= \begin{cases}\{a\}, & \text { if }|\mathfrak{b}(a) \cap X|=0, \\
\hat{0}_{\mathfrak{A}(P)}, & \text { if }|\mathfrak{b}(a) \cap X| \geq 1,\end{cases} \\
& \{a\} / X= \begin{cases}\{a\}, & \text { if }|\mathfrak{b}(a) \cap X|=0, \\
\mathfrak{b}(\mathfrak{b}(a)-X), & \text { if }|\mathfrak{b}(a) \cap X| \geq 1, \mathfrak{b}(a) \nsubseteq X, \\
\hat{1}_{\mathfrak{A}(P)}, & \text { if } \mathfrak{b}(a) \subseteq X .\end{cases}
\end{aligned}
$$

(ii) If $A$ is a nontrivial antichain of $P$ then the deletion $A \backslash X$ and contraction $A / X$ are the antichains

$$
A \backslash X=\bigvee_{a \in A}(\{a\} \backslash X), \quad A / X=\bigvee_{a \in A}(\{a\} / X)
$$

(iii) The deletion and contraction for the trivial antichains of $P$ are

$$
\begin{aligned}
& \hat{0}_{\mathfrak{H}(P)} \backslash X=\hat{0}_{\mathfrak{H}(P)} / X=\hat{0}_{\mathfrak{A}(P)}, \\
& \hat{1}_{\mathfrak{A}(P)} \backslash X=\hat{1}_{\mathfrak{H}(P)} / X=\hat{1}_{\mathfrak{A}(P)} .
\end{aligned}
$$

If we take into consideration the empty subset $\varnothing^{\mathrm{a}}$ of the atom set $P^{\mathrm{a}}$, then for every $A \in \mathfrak{A}(P)$ we define the antichains $A \backslash \varnothing^{\mathrm{a}}$ and $A / \varnothing^{\mathrm{a}}$ to be equal to $A$.

The following observations are an immediate consequence of Definition 2.1: if $\{a\}$ is a one-element antichain of $P$, then we have

$$
\begin{gathered}
\{a\} \backslash X \leq\{a\} \leq\{a\} / X, \\
\mathfrak{b}(a) \backslash X \leq \mathfrak{b}(\{a\} / X) \leq \mathfrak{b}(a) \leq \mathfrak{b}(a) / X=\mathfrak{b}(\{a\} \backslash X) .
\end{gathered}
$$

Another observation is the following lemma.

LEMMA 2.2. Let $X \subseteq P^{\mathrm{a}},|X| \geq 1$. If $A_{1}, A_{2} \in \mathcal{A}(P)$ and $A_{1} \leq A_{2}$, then

$$
A_{1} \backslash X \leq A_{2} \backslash X, \quad A_{1} / X \leq A_{2} / X
$$

If $A \in \mathfrak{A}(P)$ then the elements $A \backslash X, A$, and $A / X$ of the lattice $\mathcal{A}(P)$ are comparable. 
LEMMA 2.3. If $A \in \mathcal{H}(P)$ and $X \subseteq P^{\mathrm{a}},|X| \geq 1$, then

$$
A \backslash X \leq A \leq A / X
$$

Proof. There is nothing to prove if $A$ is a trivial antichain. Suppose that $A$ is nontrivial. With the help of (2.6) and (2.8), we see that $A \backslash X=\bigvee_{a \in A}(\{a\} \backslash X) \leq \bigvee_{a \in A}\{a\}=$ $A \leq \bigvee_{a \in A}(\{a\} / X)=A / X$.

If $\{a\}$ is a nontrivial one-element antichain of $P$ and $X \subseteq P^{\mathrm{a}},|X| \geq 1$, then we obviously have $\{a\} \backslash X=(\{a\} \backslash X) \backslash X$. The antichain $\{a\} / X$ has an analogous property. Indeed, if $|\mathfrak{b}(a) \cap X|=0$ or if $\mathfrak{b}(a) \subseteq X$ then $(\{a\} / X) / X=\{a\} / X$, due to the definition of contraction. Further, if $|\mathfrak{b}(a) \cap X| \geq 1$ and $\mathfrak{b}(a) \nsubseteq X$ then, on one hand, we have $(\{a\} / X) / X \geq\{a\} / X$, by Lemma 2.3. On the other hand, for every $b \in\{a\} / X=$ $\mathfrak{b}(\mathfrak{b}(a)-X)$ we have $\mathfrak{b}(b)-X \geq \mathfrak{b}(a)-X$ and, as a consequence, we have $(\{a\} / X) / X=$ $\bigvee_{b \in\{a\} / X}(\{b\} / X) \leq \mathfrak{b}(\mathfrak{b}(a)-X)=\{a\} / X$. We conclude that $(\{a\} / X) / X=\{a\} / X$. In view of (2.6), we can formulate the following lemma.

LEMMA 2.4. If $A \in \mathcal{H}(P)$ and $X \subseteq P^{\mathrm{a}},|X| \geq 1$, then

$$
(A \backslash X) \backslash X=A \backslash X, \quad(A / X) / X=A / X .
$$

Altogether, Lemmas 2.2, 2.3, and 2.4 describe the connection of the maps $(\backslash X)$ : $\mathcal{H}(P) \rightarrow \mathcal{H}(P)$ and $(/ X): \mathcal{H}(P) \rightarrow \mathcal{H}(P)$ with (co)closure operators (see, e.g., [1, Chapter IV]).

THEOREM 2.5. Let $X \subseteq P^{\mathrm{a}},|X| \geq 1$. The map $(\backslash X)$ is a coclosure operator on $\mathfrak{A}(P)$. The map $(/ X)$ is a closure operator on $\mathfrak{A}(P)$.

Given a nonempty atom subset $X$, we denote, slightly abusing denotations, the images $(\backslash X)(\mathcal{H}(P))=\{A \backslash X: A \in \mathcal{A}(P)\}$ and $(/ X)(\mathcal{H}(P))=\{A / X: A \in \mathcal{H}(P)\}$ by $\mathcal{H}(P) \backslash X$ and $\mathfrak{A}(P) / X$, respectively. We can interpret well-known properties of (semi)lattice maps and (co)closure operators on lattices in case of maps $(\backslash X)$ and $(/ X)$.

Definition 2.1 implies that the maps $(\backslash X),(/ X): \mathcal{H}(P) \rightarrow \mathcal{H}(P)$ are upper $\left\{\hat{0}_{\mathfrak{H}(P)}\right.$, $\left.\hat{1}_{\mathcal{A}(P)}\right\}$-homomorphisms, that is for all $A_{1}, A_{2} \in \mathcal{H}(P)$, we have $\left(A_{1} \vee A_{2}\right) \backslash X=\left(A_{1} \backslash X\right) \vee$ $\left(A_{2} \backslash X\right),\left(A_{1} \vee A_{2}\right) / X=\left(A_{1} / X\right) \vee\left(A_{2} / X\right)$ and, moreover, we have $\hat{0}_{\mathfrak{H}(P)} \backslash X=\hat{0}_{\mathfrak{H}(P)} / X=$ $\hat{0}_{\mathfrak{H}(P)}$ and $\hat{1}_{\mathcal{H}(P)} \backslash X=\hat{1}_{\mathfrak{H}(P)} / X=\hat{1}_{\mathcal{H}(P)}$.

The posets $\mathcal{A}(P) \backslash X$ and $\mathcal{A}(P) / X$, with the partial orders induced by the partial order on $\mathcal{A}(P)$, are lattices.

The lattice $\mathfrak{A}(P) \backslash X$ is a join-subsemilattice of $\mathcal{A}(P)$. Denote by $\wedge_{\mathfrak{H}(P) \backslash X}$ the operation of meet in $\mathcal{H}(P) \backslash X$. If $D_{1}, D_{2} \in \mathcal{H}(P) \backslash X$, then we have $D_{1} \wedge \mathfrak{H}(P) \backslash X D_{2}=\left(D_{1} \wedge D_{2}\right) \backslash X$.

The lattice $\mathcal{A}(P) / X$ is a sublattice of $\mathcal{A}(P)$.

If $D \in \mathcal{A}(P) \backslash X$, then the preimage $(\backslash X)^{-1}(D)$ of $D$ under the map $(\backslash X)$ is the closed interval $[D, D \vee X]$ of $\mathcal{A}(P)$.

If $D \in \mathcal{A}(P) / X$, then the preimage $(/ X)^{-1}(D)$ of $D$ under the map $(/ X)$ is a convex join-subsemilattice of the lattice $\mathcal{A}(P)$, with the greatest element $D$.

Equalities (1.4) may be generalized in the context of an arbitrary finite bounded poset. Indeed, let $A \in \mathfrak{A}(P)$ and $X \subseteq P^{\mathrm{a}},|X| \geq 1$. We can deduce from Lemma 2.3 that 
the comparisons

$$
\mathfrak{b}(A) \backslash X \leq \mathfrak{b}(A) \leq \mathfrak{b}(A) / X, \quad \mathfrak{b}(A / X) \leq \mathfrak{b}(A) \leq \mathfrak{b}(A \backslash X)
$$

hold, and we make an additional conclusion in the following theorem.

THEOREM 2.6. If $A \in \mathfrak{A}(P)$ and $X \subseteq P^{\mathrm{a}},|X| \geq 1$, then

$$
\mathfrak{b}(A) \backslash X \leq \mathfrak{b}(A / X) \leq \mathfrak{b}(A) \leq \mathfrak{b}(A) / X \leq \mathfrak{b}(A \backslash X) .
$$

Proof. There is nothing to prove if the antichain $A$ is trivial.

If $A_{1}, A_{2}$ are arbitrary antichains of $P$ and $X \subseteq P^{\mathrm{a}},|X| \geq 1$, then we can check with the help of routine machinery that

$$
\begin{aligned}
& \left(A_{1} \wedge A_{2}\right) \backslash X \leq\left(A_{1} \backslash X\right) \wedge\left(A_{2} \backslash X\right), \\
& \left(A_{1} \wedge A_{2}\right) / X \leq\left(A_{1} / X\right) \wedge\left(A_{2} / X\right) .
\end{aligned}
$$

Suppose that $A$ is nontrivial. We prove that $\mathfrak{b}(A) \backslash X \leq \mathfrak{b}(A / X)$. Using comparisons (2.15) and (2.9), we see that

$$
\mathfrak{b}(A) \backslash X=\left(\bigwedge_{a \in A} \mathfrak{b}(a)\right) \backslash X \leq \bigwedge_{a \in A}(\mathfrak{b}(a) \backslash X) \leq \bigwedge_{a \in A} \mathfrak{b}(\{a\} / X)=\mathfrak{b}\left(\bigvee_{a \in A}(\{a\} / X)\right)=\mathfrak{b}(A / X) .
$$

We prove that $\mathfrak{b}(A) / X \leq \mathfrak{b}(A \backslash X)$. With the help of (2.16) and (2.9), we see that

$$
\mathfrak{b}(A) / X=\left(\bigwedge_{a \in A} \mathfrak{b}(a)\right) / X \leq \bigwedge_{a \in A}(\mathfrak{b}(a) / X)=\bigwedge_{a \in A} \mathfrak{b}(\{a\} \backslash X)=\mathfrak{b}\left(\bigvee_{a \in A}(\{a\} \backslash X)\right)=\mathfrak{b}(A \backslash X) .
$$

\section{REFERENCES}

[1] M. Aigner, Combinatorial Theory, Grundlehren der Mathematischen Wissenschaften, vol. 234, Springer-Verlag, Berlin, 1979.

[2] R. Cordovil, K. Fukuda, and M. L. Moreira, Clutters and matroids, Discrete Math. 89 (1991), no. 2, 161-171.

[3] A. O. Matveev, On blockers in bounded posets, Int. J. Math. Math. Sci. 26 (2001), no. 10, 581-588.

[4] P. D. Seymour, The forbidden minors of binary clutters, J. London Math. Soc. (2) 12 (1976), no. 3, 356-360.

[5] R. P. Stanley, Enumerative Combinatorics. Vol. 1, Cambridge Studies in Advanced Mathematics, vol. 49, Cambridge University Press, Cambridge, 1997.

ANDRey O. MATVEeV: DATA Center Company, BoX 5, EKATERinburg 620034, Russia E-mail address: aomatveev@dc.ru 


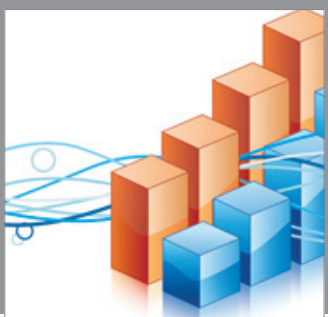

Advances in

Operations Research

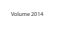

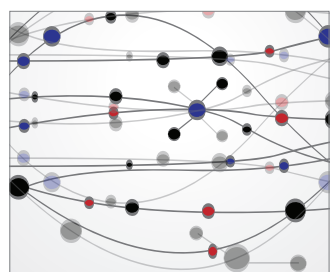

\section{The Scientific} World Journal
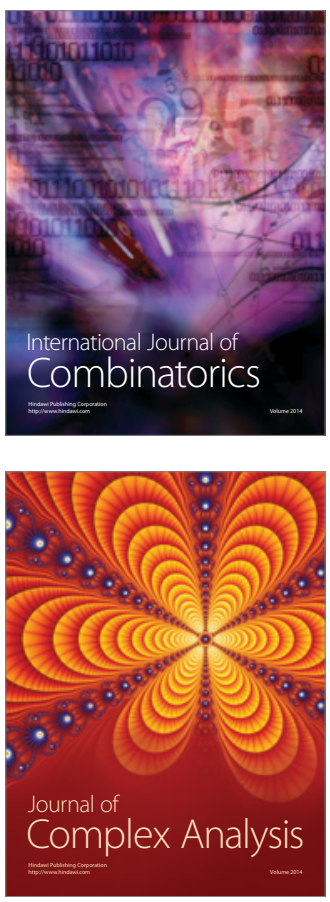

International Journal of

Mathematics and

Mathematical

Sciences
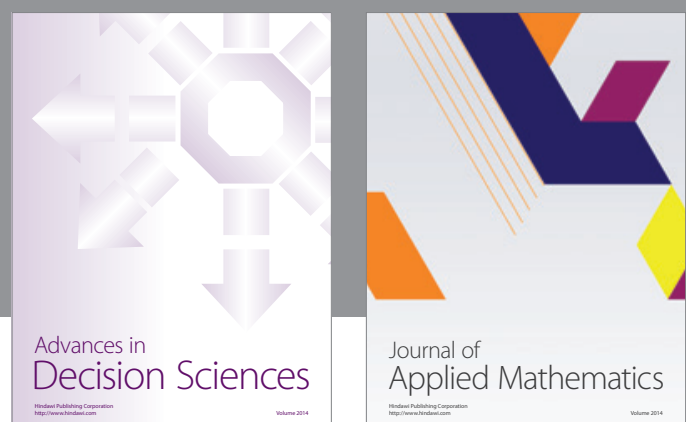

Journal of

Applied Mathematics
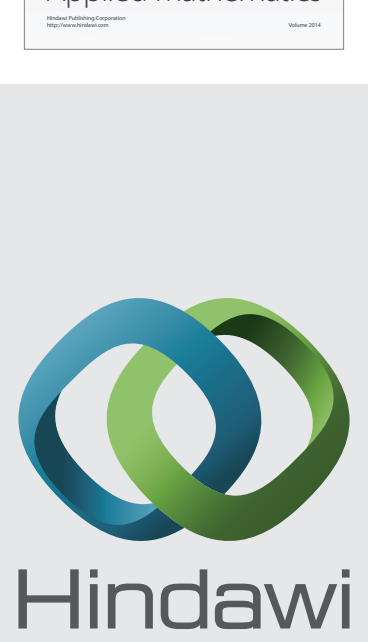

Submit your manuscripts at http://www.hindawi.com
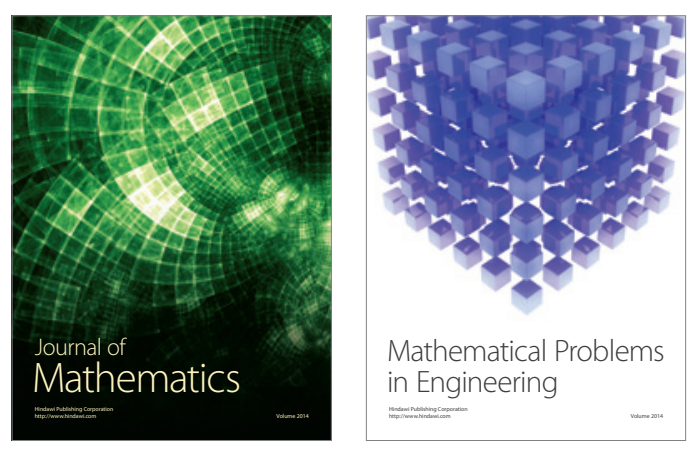

Mathematical Problems in Engineering
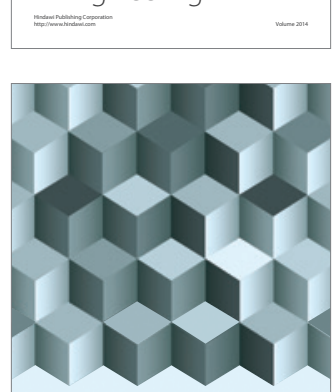

Journal of

Function Spaces
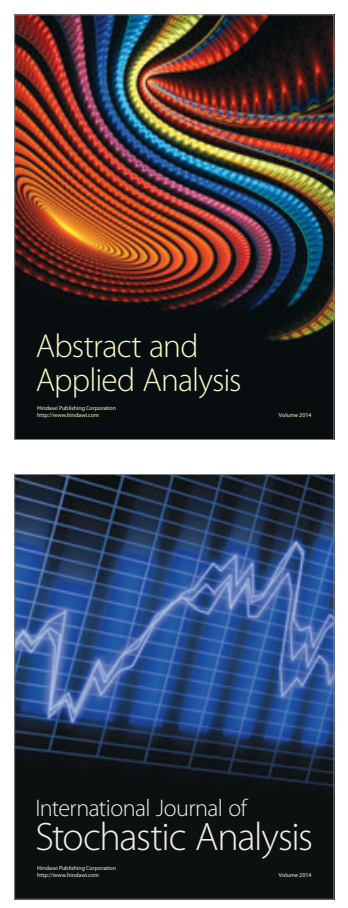

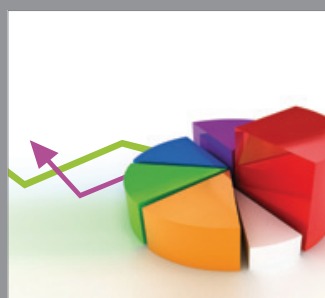

ournal of

Probability and Statistics

Promensencen
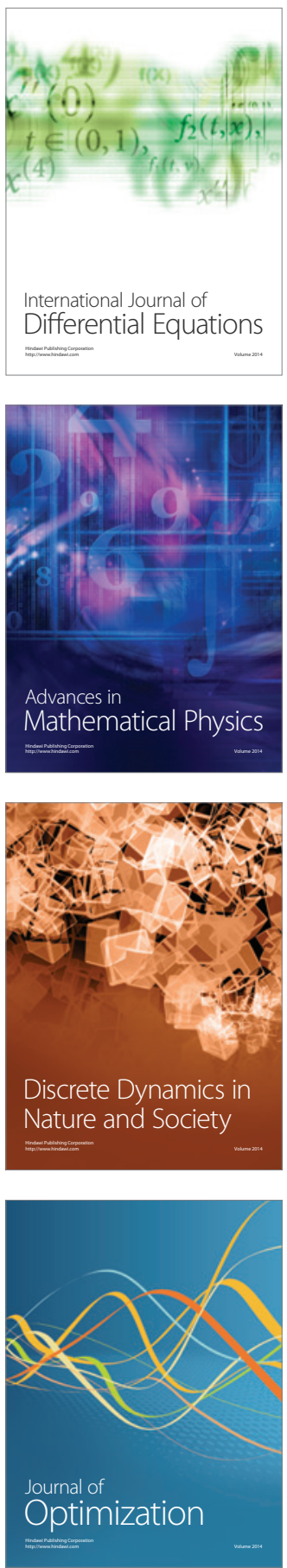